Canad. Math. Bull. Vol. 18 (1), 1975

\title{
THE EXISTENCE OF INJECTIVE EFFACEMENTS
}

\author{
BY \\ MICHAEL BARR
}

Introduction. The main result of this paper is little more than a juxtaposition of a remark in [Leroux] $(\$ 5$, part $\mathrm{A})$, with a theorem of [Banaschewski]. However, the results are individually little known and their juxtaposition not at all.

More explicitly, Leroux remarks, in somewhat different form,

THEOREM A. Let the category $\mathbf{X}$ be complete and cocomplete, and have a set of cogenerators. Then a necessary and sufficient condition that there exist injective effacements (with respect to the class of regular monomorphisms) is that $\mathbf{X}$ be coregular and satisfy, in addition, condition COEX $4^{*}$.

Banaschewski has proved, again in somewhat different form,

THEOREM B. Let the coregular category $\mathbf{X}$ be complete and cocomplete, satisfy the condition COEX 5*, and be co-well-powered. Then every object of $\mathbf{X}$ has an injective hull (with respect to the class of regular monomorphisms) if and only if there is a bound for each $X \in \mathbf{X}$ on the length of essential sequences emanating from $X$.

These terms are all defined in $\$ 1$. We remark here that in the abelian case, COEX $4 *$ and COEX $5 *$ reduce to $\mathrm{AB} 4$ and $\mathrm{AB} 5$, respectively.

Since injective effacements will allow us to bound essential sequences and $\operatorname{COEX~} 5^{*} \Rightarrow \operatorname{COEX} 4^{*}$, we are led easily to the following.

THEOREM C. Let the coregular category $\mathbf{X}$ be complete and cocomplete, have cogenerators, and satisfy condition $\mathrm{COEX} 5^{*}$. Then every object of $\mathbf{X}$ has an injective hull (with respect to the class of regular monomorphisms).

Note that Theorem $\mathrm{C}$ resembles Grothedieck's theorem for abelian categories. The only difference is that Grothedieck was able to bound essential sequences in the presence of generators, whereas here we require cogenerators. If it could be done using only generators, a very straightforward proof of the embedding theorems in [Barr] would result.

We will consistently use the notation $\mapsto$ and $\rightarrow$ to denote regular monomorphisms and epimorphisms, respectively.

Received by the editors November 10, 1972 and, in revised form, May 25, 1973. 
We use $X+{ }_{Z} Y$ to denote the pushout of the square

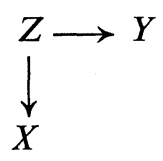

and $\Pi_{X} Y_{i}$ to denote the fibred coproduct of a family $\left\{X \rightarrow Y_{i}\right\}$.

1. Definitions. A map in a category is called a regular monomorphism if it is the equalizer of a pair of maps. If its cokernel pair exists, it is the equalizer of them. A map is called a universal regular monomorphism if its pushout with every map with the same domain exists and if the map across from it in such a pushout square is always a regular monomorphism. A category is called coregular if every regular monomorphism is universal. In that case (and provided equalizers and cokernel pairs exist), every map has a canonical factorization as an epimorphism followed by a regular monomorphism (see [Barr], I.(2.3)). A coregular category is said to satisfy condition $\operatorname{COEX} 4^{*}$ provided all coproducts exist and if, given a family of regular monomorphisms $\left\{X_{i} \mapsto Y_{i}\right\}$, the induced map $\Pi X_{i} \rightarrow \Pi Y_{i}$ is one as well. The category $\mathbf{X}$ is said to satisfy condition $C O E X 5^{*}$ if colimits exist and if, given any filtered category $\mathbf{I}$, functors $F, G: \mathbf{I} \rightarrow \mathbf{X}$, and a natural transformation $F \rightarrow G$ such that $F_{i} \mapsto G_{i}$ is a regular monomorphism for all $i \in \mathbf{I}$, then the induced map colim $F \rightarrow \operatorname{colim} G$ is one as well. A regular monomorphism $X \mapsto I$ is called an injective effacement if any diagram

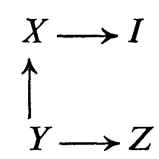

can be completed to a commutative square by a map $Z \rightarrow I$. An object $I$ is easily seen to be injective if and only if its identity map is an injective effacement. A regular monomorphism $X \mapsto Y$ is called essential if for any $Y \rightarrow Z$, if the composite $X \rightarrow Y \rightarrow Z$ is a regular monomorphism, so is $Y \rightarrow Z$. If $X \mapsto I$ is essential and $I$ is injective, then it is well known (and obvious) that $I$ is an injective hull of $X$, meaning that $I$ is a regular subobject of every injective container of $X$. A cogenerating set in $\mathbf{X}$ is a set $\Gamma$ of objects in $\mathbf{X}$ such that for every epimorphism $X \rightarrow Y$, not an isomorphism, there is a $G \in \Gamma$ and a map $X \rightarrow G$ which does not factor through $Y$.

2. Proof of Theorem A. Throughout this section $\boldsymbol{X}$ denotes a category which satisfies the hypotheses of Theorem A.

Proposition 1. Let $\mathbf{X}$ be coregular and satisfy COEX 4* and let $X \mapsto X s, s \in S$ be a family of regular monomorphisms of $\mathbf{X}$. Then the canonical map $X \rightarrow \prod_{s \in S} X_{s}$ is a regular monomorphism as well. 
Proof. First, by COEX 4*,

Second, the square

$$
\Pi X \mapsto \Pi X_{s}
$$<smiles>[Y1][Y11]([Y])([Y])[H]</smiles>

in which the top horizontal map is the codiagonal is a pushout and the result follows from coregularity.

Now we can prove the forward implication of Theorem A. Consider the set $R$ of all possible irredundant families

$$
\left\{f_{s}: X \rightarrow G_{s} \mid s \in S_{r}, r \in R, G_{s} \in \Gamma\right\},
$$

for which the induced map

$$
\left\langle f_{s}\right\rangle: X \rightarrow \prod_{s \in S_{r}} G_{s}=H_{r}
$$

is a regular monomorphism. Up to change of index sets there is only a set of such families-and a set of such $H_{r}$-since each is determined by choosing a subset $\Sigma \subset \Gamma$ and for each $G \in \Sigma$ a map $X \rightarrow G$. Finally, we let

$$
I=\prod_{r \in E} H_{r}
$$

From the preceding proposition we see that the natural map $X \mapsto I$ is a regular monomorphism. Now let $X \mapsto Y$ be any regular monomorphism. Choose a regular monomorphism $Y \mapsto \Pi_{t \in T} G_{t}$ with $G_{t} \in \Gamma$ for all $t \in T$. Then evidently $X \mapsto$ $\Pi_{t \in T} G_{t}$, but the family of $X \rightarrow G_{t}$ might be redundant. Then choose a subset $T_{0} \subset T$ so that $\left\{X \rightarrow G_{t}\right\}, t \in T_{0}$ is irredundant but representative of all the maps $X \rightarrow Y \rightarrow G_{t}$ and then $T_{0}$ is one of the $S_{r}$. Thus we have the diagram

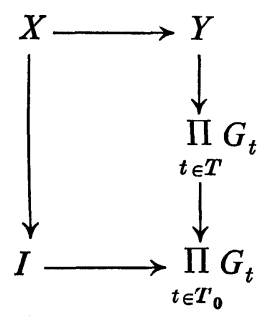

in which the bottom map is the coproduct injection. Thus any $X \mapsto Y$ gives a commutative diagram

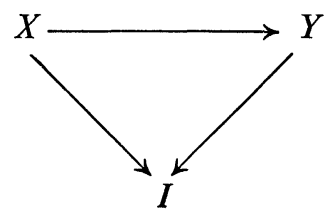


If, more generally, we have a diagram

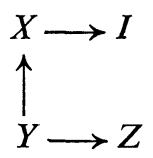

we complete it by applying the above argument to $X \mapsto X+_{Y} Z$.

The converse part of Theorem A is easy and is left to the reader. Note that in any commutative diagram

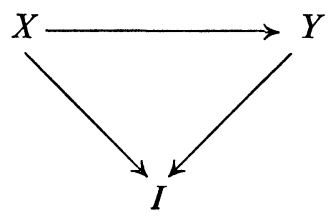

the map $X \mapsto Y$ will have to be a regular monomorphism.

3. Proof of Theorem B. Throughout this section we suppose that $\boldsymbol{X}$ satisfies the hypotheses of Theorem $\mathbf{B}$.

Proposition 2. Given any regular monomorphism $X \mapsto Y$, there is an epimorphism $Y \rightarrow Z$ such that the composite $X \rightarrow Z$ is an essential monomorphism.

Proof. If $X \mapsto Y$ is already essential, we are done. Otherwise there is a map $Y \rightarrow Z_{1}$, not a regular monomorphism, for which the composition $X \mapsto Y_{1}$ is one. Let $Y \rightarrow Y_{1} \mapsto Z_{1}$ be the epimorphism, regular monomorphism factorization of this map. Then clearly we have

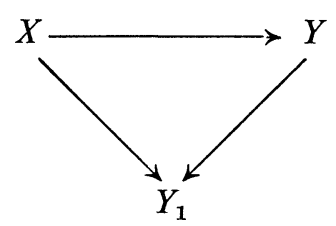

If $X \mapsto Y_{1}$ is still not essential, we can continue to have $Y_{1} \rightarrow Y_{2}$ with $X \mapsto Y_{2}$. Either the process terminates at a finite ordinal or we get a sequence

$$
X \mapsto Y \rightarrow Y_{1} \rightarrow Y_{2} \rightarrow \cdots \rightarrow Y_{n} \rightarrow \cdots
$$

such that every $X \mapsto Y_{n}$. Then by COEX 5*, we have

$$
X \mapsto Y_{\omega}=\operatorname{colim} Y_{n} .
$$

On the other hand, by general principles, $Y_{n} \rightarrow Y_{\omega}$ for all $n \in \omega$. By continuing this way, we either find an ordinal $n$ such that $X \mapsto Y_{n}$ is essential or else continue to define for all ordinals $n$ objects $Y_{n}$ and maps $f_{n}: Y \rightarrow Y_{n}, f_{m, n}: Y_{n} \rightarrow Y_{m}$ for $m \geq n$ satisfying the obvious coherence. Since $Y$ is co-well-powered there must exist $m>n$ such that $f_{n}$ and $f_{m}$ represent the same quotient object of $Y$, this means we 
have a commutative diagram

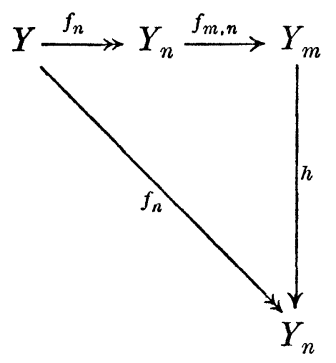

in which $h$ is an isomorphism. But the epimorphism $f_{n}$ can be cancelled from $f_{n}=h f_{m, n} f_{n}$ to give $h f_{m, n}=Y_{n}$ and $f_{m, n}=h^{-1}$ is an isomorphism. But then $f_{m, n}=f_{m, n+1} \cdot f_{n+1, n}$ is a regular monomorphism which implies (given the uniqueness of the factorization) that $f_{n+1, n}$ is and hence $f_{n+1, n}$ is an isomorphism also which contradicts its construction.

ReMARKs. This amounts to verifying the dual of $(P 3)$ in [Banaschewski]. I am indebted to the referee for pointing out a gap in my original proof of this proposition.

Now we are ready to prove the forward implication in Theorem B. Let $X$ be an object. If $X$ has an essential extension, choose one, say $X \mapsto X_{1}$. If $X_{1}$ has an essential extension, choose one $X_{1} \mapsto X_{2}$. Either the process terminates or we get a sequence, defined for all finite ordinals,

$$
X \mapsto X_{1} \mapsto X_{2} \mapsto \cdots \mapsto X_{n} \mapsto \cdots
$$

of essential extensions. Then by COEX $5 *$ the induced map

$$
X \mapsto \operatorname{colim} X_{n}
$$

is a regular monomorphism. Apply the preceding proposition to get

$$
X \mapsto \operatorname{colim} X \rightarrow X_{\omega},
$$

for which the composite is a regular monomorphism. In this way we build a transfinite sequence of essential extensions which, by hypothesis, must terminate in an object $I$ which has no essential extension. Let $I \mapsto Y$ be a regular monomorphism. Apply the proposition to get $I \mapsto Y \rightarrow Z$ with $I \mapsto Z$ an essential extension. But then $I \rightarrow Z$ is an isomorphism. Hence every regular monomorphism with domain $I$ is split. Now consider a diagram<smiles>[Y][V]</smiles>

Then $I \mapsto Y+_{X} I$ is a split monomorphism and the composite $Y \rightarrow Y+{ }_{X} I \rightarrow I$ by the splitting map gives a map $Y \rightarrow I$ which makes the triangle commute. Thus 
every object has an essential monomorphism into an injective, evidently an injective envelope.

4. Proof of Theorem C. Throughout this section we suppose that $\boldsymbol{X}$ satisfies the hypotheses of Theorem $\mathrm{C}$.

Proposition 3. The category $\mathbf{X}$ is co-well-powered.

Proof. The quotients $X \rightarrow Y$ of $X$ are determined by those maps $X \rightarrow G, G \in \Gamma$ which factor through $Y$ (see [Barr], II. (2.1)).

Proposition 4. Each object $X \in \mathbf{X}$ has only a set of regular subobjects.

Proof. A regular subobject $Y \mapsto X$ is determined by its cokernel pair $X+{ }_{Y} X$. This is a quotient of $X+X$ and there are only a set of those.

Now we can prove that a category which satisfies the hypotheses of Theorem $\mathrm{C}$ satisfies those of Theorem $\mathrm{B}$. The only thing to worry about is the possibility of a sequence of proper essential extensions

$$
X \mapsto X_{1} \mapsto X_{2} \mapsto \cdots \mapsto X_{n} \mapsto \cdots
$$

extending through all ordinals. But in that case, choose an injective effacement $X \mapsto I$. Let $N$ be the cardinal of the set of regular subobjects of $I$ and $M$ an ordinal of cardinality $>N$. Then $X_{M}$ has more than $N$ regular subobjects-all the $X_{n}$, $n<M$, at least. Now there is a commutative diagram

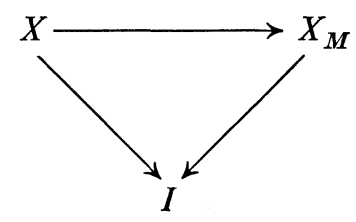

and since $X \mapsto X_{M}$ is essential, the map $X_{M^{\mapsto}} \mapsto I$ is a monomorphism as well. But then $I$ will have more than $N$ subobjects, a contradiction.

REMARK. The referee has observed that in order to find an injective hull of a single $X$, it is not necessary to suppose all coproducts exist; only those up to $\sum_{G_{\epsilon \Gamma}} \#(X, G)$ are required.

\section{REFERENCES}

1. B. Banaschewski, Projective covers in categories of topological spaces and topological algebras, "Proceedings of the Topology Conference", Kanpur, 1968, (to appear).

2. M. Barr, Exact categories, in "Exact Categories and Categories of Sheaves", Lecture Notes in Mathematics 236 (1971), 1-120.

3. P. Leroux, Sur les structures d'effacement, Math. Z. 121 (1971), 329-340.

Department of Mathematics

MCGILL UNIVERSITY

P.O. BOX 6070

Montreal 101, CANADA 